\title{
Transcendence of stammering continued fractions
}

\author{
Yann Bugeaud
}

To the memory of Alf van der Poorten

\begin{abstract}
Let $\theta=\left[0 ; a_{1}, a_{2}, \ldots\right]$ be an algebraic number of degree at least three. Recently, we have established that the sequence of partial quotients $\left(a_{\ell}\right)_{\ell \geq 1}$ of $\theta$ is not too simple and cannot be generated by a finite automaton. In this expository paper, we point out the main ingredients of the proof and we briefly survey earlier works.
\end{abstract}

\section{Introduction}

It is widely believed that the continued fraction expansion of an irrational algebraic number

$$
\theta=\lfloor\theta\rfloor+\left[0 ; a_{1}, a_{2}, \ldots, a_{\ell}, \ldots\right]=\lfloor\theta\rfloor+\frac{1}{a_{1}+\frac{1}{a_{2}+\frac{1}{\ldots}}}
$$

either is eventually periodic (and we know that this is the case if, and only if, $\theta$ is a quadratic irrational), or contains arbitrarily large partial quotients. Here, and in all what follows, $\lfloor x\rfloor$ and $\lceil x\rceil$ denote, respectively, the integer part and the upper integer part of the real number $x$.

A preliminary step consists in providing explicit examples of transcendental continued fractions. The first result of this type goes back to Liouville [16], who constructed transcendental real numbers with a very fast growing sequence of partial quotients. His key tool is the so-called Liouville inequality which asserts that, if $\theta$ is a real algebraic number of degree $d \geq 2$, then there exists a positive constant $c_{1}(\theta)$ such that

$$
|\theta-p / q| \geq c_{1}(\theta) q^{-d}, \quad \text { for every rational number } p / q \text { with } q \geq 1 \text {. }
$$

Subsequently, various authors used deeper transcendence criteria from Diophantine approximation to construct other classes of transcendental continued fractions. Of particular

2000 Mathematics Subject Classification : 11J70, 11J81, 11J87. Keywords: continued fractions, transcendence. 
interest is the work of Maillet [19] (see also Section 34 of [22]), who was the first to give explicit examples of transcendental continued fractions with bounded partial quotients. A particular case of Maillet's result asserts that if $\left(a_{\ell}\right)_{\ell \geq 1}$ is a non-eventually periodic sequence of positive integers at most equal to $M$, and if there is an increasing sequence $\left(\ell_{n}\right)_{n \geq 1}$ such that

$$
a_{\ell_{n}}=a_{\ell_{n}+1}=\ldots=a_{n \ell_{n}}=1,
$$

for $n \geq 1$, then the real number

$$
\alpha=\left[0 ; a_{1}, a_{2}, \ldots\right]
$$

is transcendental. His proof is based on a general form of the Liouville inequality which limits the approximation of real algebraic numbers $\theta$ of degree $d \geq 3$ by quadratic irrationals. More precisely, Maillet showed that there exists a positive constant $c_{2}(\theta)$ such that

$$
|\theta-\gamma| \geq c_{2}(\theta) H(\gamma)^{-d}, \quad \text { for every real quadratic number } \gamma
$$

Here, and everywhere in the present text, $H(P)$ denotes the height of the integer polynomial $P(X)$, that is, the maximum of the absolute values of its coefficients; furthermore, $H(\gamma)$ denotes the height of the algebraic number $\gamma$, that is, the height of its minimal defining polynomial over $\mathbf{Z}$. A rapid (and rough) calculation shows that the height of the quadratic irrational real number

$$
\alpha_{n}:=\left[0 ; a_{1}, \ldots, a_{\ell_{n}-1}, \overline{1}\right]
$$

where the notation $\overline{1}$ means that the partial quotient 1 is repeated infinitely many times, satisfies

$$
H\left(\alpha_{n}\right) \leq \prod_{i=1}^{\ell_{n}}\left(a_{i}+2\right)^{2} \leq(M+2)^{2 \ell_{n}} .
$$

This provides us with infinitely many very good approximations to $\alpha$. Indeed, by construction, for $n \geq 1$, the first $n \ell_{n}$ partial quotients of $\alpha$ and $\alpha_{n}$ are the same, thus we derive from (1.2) and (4.4) below that

$$
\left|\alpha-\alpha_{n}\right|<2^{2-n \ell_{n}} \leq 4 H\left(\alpha_{n}\right)^{-n(\log 2) /(2 \log (M+2))} .
$$

It then follows from (1.1) that $\alpha$ cannot be algebraic of degree $\geq 3$. As $\left(a_{\ell}\right)_{\ell \geq 1}$ is infinite and not eventually periodic, $\alpha$ is transcendental.

A. Baker [9] used in 1962 Roth's theorem for number fields obtained by LeVeque to strongly improve upon the results of Maillet and make them more explicit. He observed that, when infinitely many of the quadratic approximations found by Maillet lie in the same quadratic number field, one can replace the use of (1.1) by that of LeVeque's Theorem, which asserts that, for any given real number field $K$, any positive real number $\varepsilon$, and any real algebraic number $\theta$ lying outside $K$, there exists a positive constant $c_{3}(\theta, \varepsilon)$ such that

$$
|\theta-\gamma| \geq c_{3}(\theta, \varepsilon) H(\gamma)^{-2-\varepsilon}, \quad \text { for every real algebraic number } \gamma \text { in } K \text {. }
$$


This is clearly relevant for the example mentioned above, since all the $\alpha_{n}$ belong to the quadratic field $\mathbf{Q}(\sqrt{5})$. In particular, it follows from (1.3) and (1.4) that if $\left(a_{\ell}\right)_{\ell \geq 1}$ is a non-eventually periodic sequence of positive integers at most equal to $M$, and if there is an increasing sequence $\left(\ell_{n}\right)_{n \geq 1}$ such that

$$
a_{\ell_{n}}=a_{\ell_{n}+1}=\ldots=a_{\left\lfloor\kappa \ell_{n}\right\rfloor}=1
$$

for $n \geq 1$ and some real number $\kappa>4(\log (M+2)) /(\log 2)$, then the real number $\alpha=$ $\left[0 ; a_{1}, a_{2}, \ldots\right]$ is transcendental.

Subsequently, further transcendence results have been obtained by applying a corollary to the Schmidt Subspace Theorem which states that, for any positive real number $\varepsilon$ and any real algebraic number $\theta$ of degree at least 3 , there exists a positive constant $c_{4}(\theta, \varepsilon)$ such that

$$
|\theta-\gamma| \geq c_{4}(\theta, \varepsilon) H(\gamma)^{-3-\varepsilon}, \quad \text { for every real quadratic number } \gamma
$$

see Corollary 3.2 below. The difference between (1.4) and (1.5) is that one takes into account every real quadratic number in (1.5), while the approximants in (1.4) all belong to the same number field. By means of (1.5), Davison [14], Queffélec [23] and other authors $[15,7]$ established the transcendence of several families of continued fractions with bounded partial quotients. In particular, the real number whose sequence of partial quotients is the Thue-Morse sequence or any Sturmian or quasi-Sturmian sequence is transcendental [23, 7].

The next step, initiated in [1], has been the use of the Schmidt Subspace Theorem, instead of its corollary (1.5), to get several combinatorial transcendence criteria for continued fraction expansions $[1,3,4,5]$. Recently in [10], we have shown how a slight modification of their proofs allows us to considerably improve two of these criteria. In the present survey, we focus on the new combinatorial transcendence criterion for stammering continued fractions established in [10] and explain the two main ingredients of its proof. We also point out some of its applications, including that to the Cobham-Loxton-van der Poorten conjecture for automatic continued fraction expansions.

\section{Recent results}

Throughout this note, we identify a sequence $\mathbf{a}=\left(a_{\ell}\right)_{\ell \geq 1}$ of positive integers with the infinite word $a_{1} a_{2} \ldots a_{\ell} \ldots$, as well denoted by $\mathbf{a}$. This should not cause any confusion.

For $n \geq 1$, we denote by $p(n, \mathbf{a})$ the number of distinct blocks of $n$ consecutive letters occurring in the word $\mathbf{a}$, that is,

$$
p(n, \mathbf{a}):=\operatorname{Card}\left\{a_{\ell+1} \ldots a_{\ell+n}: \ell \geq 0\right\} .
$$

The function $n \mapsto p(n, \mathbf{a})$ is called the complexity function of $\mathbf{a}$. A well-known result of Morse and Hedlund $[20,21]$ asserts that $p(n, \mathbf{a}) \geq n+1$ for $n \geq 1$, unless a is ultimately periodic (in which case there exists a constant $C$ such that $p(n, \mathbf{a}) \leq C$ for $n \geq 1$ ). 
Let $\alpha$ be an irrational real number and write

$$
\alpha=\lfloor\alpha\rfloor+\left[0 ; a_{1}, a_{2}, \ldots\right] .
$$

Let a denote the infinite word $a_{1} a_{2} \ldots$ A natural way to measure the intrinsic complexity of $\alpha$ is to count the number $p(n, \alpha):=p(n, \mathbf{a})$ of distinct blocks of given length $n$ in the word a.

Let $\alpha$ be a real algebraic number of degree at least three. A first step towards a proof that $\alpha$ has unbounded partial quotients would be to get a good lower bound for $p(n, \alpha)$. Theorem 1.1 of [10], reproduced below, asserts that the complexity function of an algebraic number of degree at least three cannot increase too slowly.

Theorem 2.1. Let $\mathbf{a}=\left(a_{\ell}\right)_{\ell \geq 1}$ be a sequence of positive integers which is not ultimately periodic. If the real number

$$
\alpha:=\left[0 ; a_{1}, a_{2}, \ldots, a_{\ell}, \ldots\right]
$$

is algebraic, then

$$
\lim _{n \rightarrow+\infty} \frac{p(n, \alpha)}{n}=+\infty
$$

Theorem 2.1 improves Theorem 7 from [7] and Theorem 4 from [1], where

$$
\lim _{n \rightarrow+\infty} p(n, \alpha)-n=+\infty
$$

was proved instead of (2.1). This gives a positive answer to Problem 3 of [1].

An infinite sequence $\mathbf{a}=\left(a_{\ell}\right)_{\ell>1}$ is an automatic sequence if it can be generated by a finite automaton, that is, if there exists an integer $k \geq 2$ such that $a_{\ell}$ is a finitestate function of the representation of $\ell$ in base $k$, for every $\ell \geq 1$. We refer the reader to [8] for a more precise definition and examples of automatic sequences. Let $b \geq 2$ be an integer. In 1968, Cobham [12] asked whether a real number whose $b$-ary expansion can be generated by a finite automaton is always either rational or transcendental. After several attempts by Cobham himself and by Loxton and van der Poorten [17], Loxton and van der Poorten [18] asserted in 1988 that the $b$-ary expansion of an irrational algebraic number cannot be generated by a finite automaton. The proof proposed in [18], which rests on a method introduced by Mahler, contains unfortunately a gap. A positive answer to Cobham's question was finally given in [2], by means of the combinatorial transcendence criterion established in [6]. Since the complexity function of every automatic sequence a satisfies $p(n, \mathbf{a})=O(n)$ (this was proved by Cobham [13] in 1972), Theorem 2.1 implies straightforwardly the next result.

Theorem 2.2. The continued fraction expansion of an algebraic number of degree at least three cannot be generated by a finite automaton.

Before stating our combinatorial transcendence criterion for continued fractions, we introduce some notation. The length of a word $W$, that is, the number of letters composing $W$, is denoted by $|W|$. For any positive integer $k$, we write $W^{k}$ for the word $W \ldots W$ 
( $k$ times repeated concatenation of the word $W$ ). More generally, for any positive real number $x$, we denote by $W^{x}$ the word $W^{\lfloor x\rfloor} W^{\prime}$, where $W^{\prime}$ is the prefix of $W$ of length $\lceil(x-\lfloor x\rfloor)|W|\rceil$.

Let $\mathbf{a}=\left(a_{\ell}\right)_{\ell \geq 1}$ be a sequence of positive integers. We say that a satisfies Condition

(*) if $\mathbf{a}$ is not ultimately periodic and if there exist $w>1$ and two sequences of finite words $\left(U_{n}\right)_{n \geq 1},\left(V_{n}\right)_{n \geq 1}$ such that:

(i) for every $n \geq 1$, the word $U_{n} V_{n}^{w}$ is a prefix of the word a;

(ii) the sequence $\left(\left|U_{n}\right| /\left|V_{n}\right|\right)_{n \geq 1}$ is bounded;

(iii) the sequence $\left(\left|V_{n}\right|\right)_{n \geq 1}$ is increasing.

Equivalently, the word a satisfies Condition $(*)$ if there exists a positive real number $\varepsilon$ such that, for arbitrarily large integers $N$, the prefix $a_{1} a_{2} \ldots a_{N}$ of a contains two disjoint occurrences of a word of length $\lfloor\varepsilon N\rfloor$.

The key tool for the proofs of Theorems 2.1 and 2.2 is the following combinatorial transcendence criterion.

Theorem 2.3. Let $\mathbf{a}=\left(a_{\ell}\right)_{\ell \geq 1}$ be a sequence of positive integers. Let $\left(p_{\ell} / q_{\ell}\right)_{\ell \geq 1}$ denote the sequence of convergents to the real number

$$
\alpha:=\left[0 ; a_{1}, a_{2}, \ldots, a_{\ell}, \ldots\right] .
$$

Assume that the sequence $\left(q_{\ell}^{1 / \ell}\right)_{\ell \geq 1}$ is bounded. If a satisfies Condition $(*)$, then $\alpha$ is transcendental.

Theorem 2.3 was established in [10]. Its proof uses the Schmidt Subspace Theorem; see Theorem 3.1 below. Consequently, the proofs of Theorems 2.1 and 2.2 rest ultimately on the Schmidt Subspace Theorem. This is also the case for the similar results on expansions of irrational algebraic numbers to an integer base; see $[2,6]$.

A simple combinatorial study (see e.g. [10]) shows that if (2.1) does not hold for a real number $\alpha:=\left[0 ; a_{1}, a_{2}, \ldots, a_{\ell}, \ldots\right]$, then the sequence $\left(a_{\ell}\right)_{\ell \geq 1}$ either is ultimately periodic, or satisfies Condition $(*)$ above. In the latter case, Theorem 2.3 implies that $\alpha$ is transcendental. This shows that Theorem 2.1 is a consequence of Theorem 2.3.

\section{The Schmidt Subspace Theorem}

The proof of Theorem 2.3 rests on the Schmidt Subspace Theorem.

Theorem 3.1 (W. M. Schmidt). Let $m \geq 2$ be an integer. Let $L_{1}, \ldots, L_{m}$ be linearly independent linear forms in $\mathbf{x}=\left(x_{1}, \ldots, x_{m}\right)$ with algebraic coefficients. Let $\varepsilon$ be a positive real number. Then, the set of solutions $\mathbf{x}=\left(x_{1}, \ldots, x_{m}\right)$ in $\mathbf{Z}^{m}$ to the inequality

$$
\left|L_{1}(\mathbf{x}) \cdots L_{m}(\mathbf{x})\right| \leq\left(\max \left\{\left|x_{1}\right|, \ldots,\left|x_{m}\right|\right\}\right)^{-\varepsilon}
$$

lies in finitely many proper subspaces of $\mathbf{Q}^{m}$.

Proof. See e.g. [25, 26]. 
Roth's theorem (that is, (1.4) with $\mathbf{K}=\mathbf{Q}$ ) is equivalent to the case $m=2$ of Theorem 3.1. We point out an immediate consequence of the case $m=3$ of Theorem 3.1, which extends Roth's theorem to approximation by quadratic numbers.

Corollary 3.2. Let $\theta$ be a real algebraic number of degree at least 3 . Let $\varepsilon$ be a positive real number. Then, there are only finitely many integer polynomials $P(X)$ of degree at most 2 such that

$$
|P(\theta)|<H(P)^{-2-\varepsilon}
$$

Consequently, there exists a positive constant $c(\theta, \varepsilon)$ such that

$$
|\theta-\gamma|>c(\theta, \varepsilon) H(\gamma)^{-3-\varepsilon}
$$

for any algebraic number $\gamma$ of degree at most 2 .

Proof. By Theorem 3.1 applied with the three linear forms $X_{2} \theta^{2}+X_{1} \theta+X_{0}, X_{1}, X_{2}$, the set of integer triples $\left(x_{0}, x_{1}, x_{2}\right)$ satisfying

$$
\left|x_{2} \theta^{2}+x_{1} \theta+x_{0}\right| \cdot\left|x_{1}\right| \cdot\left|x_{2}\right| \leq\left(\max \left\{\left|x_{0}\right|,\left|x_{1}\right|,\left|x_{2}\right|\right\}\right)^{-\varepsilon}
$$

lies in finitely many proper subspaces of $\mathbf{Q}^{3}$. If $x_{1} x_{2}=0$, then, by Roth's theorem (that is, (1.4) for $\mathbf{K}=\mathbf{Q})$, there are only finitely many integers $y_{0}, y_{2}, z_{0}, z_{1}$ such that $y_{2} z_{1} \neq 0$ and

$$
\left|y_{2}\right| \cdot\left|y_{2} \theta^{2}+y_{0}\right|<\left(\max \left\{\left|y_{0}\right|,\left|y_{2}\right|\right\}\right)^{-\varepsilon}, \quad\left|z_{1}\right| \cdot\left|z_{1} \theta+z_{0}\right|<\left(\max \left\{\left|z_{0}\right|,\left|z_{1}\right|\right\}\right)^{-\varepsilon} .
$$

Consequently, we can assume that $x_{1}$ and $x_{2}$ are both non-zero.

Let $a_{0} X_{0}+a_{1} X_{1}+a_{2} X_{2}=0$ denote a proper subspace of $\mathbf{Q}^{3}$, with $a_{0}, a_{1}, a_{2}$ in $\mathbf{Z}$ and $a_{0} \neq 0$. If (3.1) and $a_{0} x_{0}+a_{1} x_{1}+a_{2} x_{2}=0$ hold for an integer triple $\left(x_{0}, x_{1}, x_{2}\right)$ with $x_{1} x_{2} \neq 0$, then

$$
\left|x_{2} \theta^{2}+x_{1} \theta+x_{0}\right|=\left|x_{2}\left(\theta^{2}-a_{2} / a_{0}\right)+x_{1}\left(\theta-a_{1} / a_{0}\right)\right|
$$

By Roth's theorem, there are only finitely many integer pairs $\left(x_{1}, x_{2}\right)$ such that $x_{1} x_{2} \neq 0$ and

$$
\left|x_{2}\left(\theta^{2}-a_{2} / a_{0}\right)+x_{1}\left(\theta-a_{1} / a_{0}\right)\right| \cdot\left|x_{1}\right| \cdot\left|x_{2}\right| \leq\left(\max \left\{\left|x_{1}\right|,\left|x_{2}\right|\right\}\right)^{-\varepsilon}
$$

Consequently, the triple $\left(x_{0}, x_{1}, x_{2}\right)$ is lying in a finite set, which depends on $a_{0}, a_{1}, a_{2}$. This proves the first statement of the corollary.

The second statement follows immediately since there is an absolute constant $c$ such that, for any integer polynomial $P(X)$, we have

$$
|P(\theta)| \leq c H(P) \cdot|\theta-\gamma|
$$

where $\gamma$ is the root of $P(X)$ which is the closest to $\theta$.

\section{Auxiliary results on continued fractions}


Classical references on the theory of continued fractions include [22, 26].

Let $\alpha:=\left[0 ; a_{1}, a_{2}, \ldots\right]$ be a real irrational number. Set $p_{-1}=q_{0}=1$ and $q_{-1}=p_{0}=0$. For $\ell \geq 1$, set $p_{\ell} / q_{\ell}=\left[0 ; a_{1}, a_{2}, \ldots, a_{\ell}\right]$ and note that

$$
q_{\ell}=a_{\ell} q_{\ell-1}+q_{\ell-2} .
$$

The theory of continued fraction implies that

$$
\left|q_{\ell} \alpha-p_{\ell}\right|<q_{\ell+1}^{-1}, \quad \text { for } \ell \geq 1,
$$

and

$$
q_{\ell+h} \geq q_{\ell}(\sqrt{2})^{h-1}, \quad \text { for } h, \ell \geq 1 .
$$

It follows from (4.1) that, if two real irrational numbers $\alpha$ and $\alpha^{\prime}$ have the same first $\ell$ partial quotients for some integer $\ell \geq 1$, then

$$
\left|\alpha-\alpha^{\prime}\right| \leq 2 q_{\ell}^{-2}
$$

where $q_{\ell}$ denotes the denominator of the $\ell$-th convergent to $\alpha$, and

$$
\left|\alpha-\alpha^{\prime}\right| \leq 2^{2-\ell}
$$

by (4.2).

In this and the next sections, we use the notation

$$
\left[0 ; a_{1}, \ldots, a_{r}, \overline{a_{r+1}, \ldots, a_{r+s}}\right]:=[0 ; U, \bar{V}],
$$

where $U=a_{1} \ldots a_{r}$ and $V=a_{r+1} \ldots a_{r+s}$, to indicate that the block of partial quotients $a_{r+1}, \ldots, a_{r+s}$ is repeated infinitely many times. We also denote by $\zeta^{\prime}$ the Galois conjugate of a quadratic real number $\zeta$. We reproduce below Lemma 6.1 from [11].

Lemma 4.1. Let $\alpha$ be a quadratic real number with ultimately periodic continued fraction expansion

$$
\alpha=\left[0 ; a_{1}, \ldots, a_{r}, \overline{a_{r+1}, \ldots, a_{r+s}}\right],
$$

with $r \geq 3$ and $s \geq 1$, and denote by $\alpha^{\prime}$ its Galois conjugate. Let $\left(p_{\ell} / q_{\ell}\right)_{\ell \geq 1}$ denote the sequence of convergents to $\alpha$. There exists an absolute constant $\kappa$ such that, if $a_{r} \neq a_{r+s}$, then we have

$$
\left|\alpha-\alpha^{\prime}\right| \leq \kappa a_{r}^{2} \max \left\{a_{r-2}, a_{r-1}\right\} q_{r}^{-2} .
$$

Lemma 4.1 is an easy consequence of the theorem of Galois (see [22], page 83) which states that the Galois conjugate of

$$
\left[a_{r+1} ; \overline{a_{r+2}, \ldots, a_{r+s}, a_{r+1}}\right]
$$

is the quadratic number

$$
\left[0 ; \overline{a_{r+s}, \ldots, a_{r+2}, a_{r+1}}\right] .
$$

Although we do not use it in the computation (5.9) below, it can be considered as a key observation for the proof of Theorem 2.3. 


\section{Transcendence criterion for stammering continued fractions}

In this section, we explain the main ingredients of the proof of Theorem 2.3. Let $\mathbf{a}=\left(a_{\ell}\right)_{\ell \geq 1}$ be a sequence of positive integers. Let $w$ and $w^{\prime}$ be non-negative real numbers with $w>1$. We say that a satisfies Condition $(*)_{w, w^{\prime}}$ if $\mathbf{a}$ is not ultimately periodic and if there exist two sequences of finite words $\left(U_{n}\right)_{n \geq 1},\left(V_{n}\right)_{n \geq 1}$ such that:

(i) for every $n \geq 1$, the word $U_{n} V_{n}^{w}$ is a prefix of the word $\mathbf{a}$;

(ii) the sequence $\left(\left|U_{n}\right| /\left|V_{n}\right|\right)_{n \geq 1}$ is bounded from above by $w^{\prime}$;

(iii) the sequence $\left(\left|V_{n}\right|\right)_{n \geq 1}$ is increasing.

Theorem 5.1. Let $\mathbf{a}=\left(a_{\ell}\right)_{\ell \geq 1}$ be a sequence of positive integers. Let $\left(p_{\ell} / q_{\ell}\right)_{\ell \geq 1}$ denote the sequence of convergents to the real number

$$
\alpha:=\left[0 ; a_{1}, a_{2}, \ldots, a_{\ell}, \ldots\right]
$$

Assume that the sequence $\left(q_{\ell}^{1 / \ell}\right)_{\ell \geq 1}$ is bounded. If there exist non-negative real numbers $w$ and $w^{\prime}$ with $w>1$ such that a satisfies Condition $(*)_{w, w^{\prime}}$, then $\alpha$ is transcendental.

Theorem 5.1, established in [10], improves Theorem 2 from [1] and Theorem 3.1 from [5], where the assumption

$$
w>((2 \log M / \log m)-1) w^{\prime}+1
$$

was required, with $M=\lim \sup _{\ell \rightarrow+\infty} q_{\ell}^{1 / \ell}$ and $m=\liminf _{\ell \rightarrow+\infty} q_{\ell}^{1 / \ell}$. Furthermore, it contains Theorem 3.2 from [3].

The reader is directed to [10] for a complete proof of Theorem 5.1. We content ourselves to explain how Theorem 3.1 and Corollary 3.2 can be applied to prove the transcendence of families of stammering continued fractions. We compare the various results obtained under the assumption that the sequence $\left(q_{\ell}^{1 / \ell}\right)_{\ell \geq 1}$ converges, which makes the comparisons easier.

Assume that the real numbers $w$ and $w^{\prime}$ are fixed, as well as the sequences $\left(U_{n}\right)_{n \geq 1}$ and $\left(V_{n}\right)_{n \geq 1}$ occurring in the definition of Condition $(*)_{w, w^{\prime}}$. Set $r_{n}=\left|U_{n}\right|$ and $s_{n}=\left|V_{n}\right|$, for $n \geq 1$. We assume that the real number $\alpha:=\left[0 ; a_{1}, a_{2}, \ldots\right]$ is algebraic of degree at least three. Throughout this section, the numerical constants implied in $\ll$ are absolute.

We observe that $\alpha$ admits infinitely many good quadratic approximants obtained by truncating its continued fraction expansion and completing by periodicity. With the above notation, for $n \geq 1$, the real number $\alpha$ is close to the quadratic number

$$
\alpha_{n}=\left[0 ; U_{n}, \overline{V_{n}}\right] .
$$

Namely, since the first $r_{n}+\left\lfloor w s_{n}\right\rfloor$ partial quotients of $\alpha$ and of $\alpha_{n}$ are the same, we deduce from (4.3) that

$$
\left|\alpha-\alpha_{n}\right| \leq 2 q_{r_{n}+\left\lfloor w s_{n}\right\rfloor}^{-2} .
$$


Furthermore, $\alpha_{n}$ is root of the quadratic polynomial (see e.g. [22])

$$
\begin{aligned}
P_{n}(X): & =\left(q_{r_{n}-1} q_{r_{n}+s_{n}}-q_{r_{n}} q_{r_{n}+s_{n}-1}\right) X^{2} \\
& -\left(q_{r_{n}-1} p_{r_{n}+s_{n}}-q_{r_{n}} p_{r_{n}+s_{n}-1}+p_{r_{n}-1} q_{r_{n}+s_{n}}-p_{r_{n}} q_{r_{n}+s_{n}-1}\right) X \\
& +\left(p_{r_{n}-1} p_{r_{n}+s_{n}}-p_{r_{n}} p_{r_{n}+s_{n}-1}\right),
\end{aligned}
$$

and we deduce that

$$
H\left(\alpha_{n}\right) \leq H\left(P_{n}\right) \leq 2 q_{r_{n}} q_{r_{n}+s_{n}}
$$

Consequently,

$$
\left|\alpha-\alpha_{n}\right| \ll H\left(\alpha_{n}\right)^{-2\left(\log q_{r_{n}+\left\lfloor w s_{n}\right\rfloor}\right) /\left(\log q_{r_{n}} q_{r_{n}+s_{n}}\right)} .
$$

Assuming that $\left(q_{\ell}^{1 / \ell}\right)_{\ell \geq 1}$ converges, our assumption that $\alpha$ is algebraic contradicts the last assertion of Corollary 3.2 when

$$
\limsup _{n \rightarrow+\infty} \frac{r_{n}+\left\lfloor w s_{n}\right\rfloor}{2 r_{n}+s_{n}}>\frac{3}{2}
$$

that is, when

$$
w>2 w^{\prime}+3 / 2 \text {. }
$$

This is the approach followed in $[23,7]$. It applies for instance when $\left(a_{\ell}\right)_{\ell \geq 1}$ is the ThueMorse sequence

$$
\mathbf{t}:=1221211221121221 \ldots
$$

on $\{1,2\}$ defined as the fixed point beginning by 1 of the morphism $\tau$ defined by $\tau(1)=12$ and $\tau(2)=21$. Indeed, for each $n \geq 1$, the prefix of length $5 \cdot 2^{n}$ of $\mathbf{t}$ is equal to its prefix of length $3 \cdot 2^{n}$ raised to the power $5 / 3$. Thus, we can take $w=5 / 3$ and $w^{\prime}=0$. The fact that the sequence $\left(q_{\ell}^{1 / \ell}\right)_{\ell \geq 1}$ converges in this case has been established in [24].

The main new ingredient in [1] is the use of Theorem 3.1 with $m=4$, instead of Corollary 3.2, which is deduced from Theorem 3.1 with $m=3$. Let us now explain to which system of four linear forms we apply Theorem 3.1. By (4.1), we have

$$
\begin{aligned}
& \left|\left(q_{r_{n}-1} q_{r_{n}+s_{n}}-q_{r_{n}} q_{r_{n}+s_{n}-1}\right) \alpha-\left(q_{r_{n}-1} p_{r_{n}+s_{n}}-q_{r_{n}} p_{r_{n}+s_{n}-1}\right)\right| \\
& \quad \leq q_{r_{n}-1}\left|q_{r_{n}+s_{n}} \alpha-p_{r_{n}+s_{n}}\right|+q_{r_{n}}\left|q_{r_{n}+s_{n}-1} \alpha-p_{r_{n}+s_{n}-1}\right| \\
& \quad \leq 2 q_{r_{n}} q_{r_{n}+s_{n}}^{-1}
\end{aligned}
$$

and, likewise,

$$
\begin{aligned}
& \left|\left(q_{r_{n}-1} q_{r_{n}+s_{n}}-q_{r_{n}} q_{r_{n}+s_{n}-1}\right) \alpha-\left(p_{r_{n}-1} q_{r_{n}+s_{n}}-p_{r_{n}} q_{r_{n}+s_{n}-1}\right)\right| \\
& \quad \leq q_{r_{n}+s_{n}}\left|q_{r_{n}-1} \alpha-p_{r_{n}-1}\right|+q_{r_{n}+s_{n}-1}\left|q_{r_{n}} \alpha-p_{r_{n}}\right| \\
& \quad \leq 2 q_{r_{n}}^{-1} q_{r_{n}+s_{n}} .
\end{aligned}
$$

Furthermore, we have

$$
\left|P_{n}(\alpha)\right| \ll H\left(P_{n}\right) \cdot\left|\alpha-\alpha_{n}\right| \ll q_{r_{n}} q_{r_{n}+s_{n}} q_{r_{n}+\left\lfloor w s_{n}\right\rfloor}^{-2} .
$$


We consider the four linearly independent linear forms

$$
\begin{aligned}
& L_{1}\left(X_{1}, X_{2}, X_{3}, X_{4}\right)=\alpha^{2} X_{1}-\alpha\left(X_{2}+X_{3}\right)+X_{4}, \\
& L_{2}\left(X_{1}, X_{2}, X_{3}, X_{4}\right)=\alpha X_{1}-X_{2}, \\
& L_{3}\left(X_{1}, X_{2}, X_{3}, X_{4}\right)=\alpha X_{1}-X_{3}, \\
& L_{4}\left(X_{1}, X_{2}, X_{3}, X_{4}\right)=X_{1} .
\end{aligned}
$$

Instead of treating the coefficient of $X$ in $P_{n}(X)$ as a single variable, we cut it into two variables. Evaluating these linear forms on the quadruple

$$
\begin{array}{r}
\underline{z}_{n}:=\left(q_{r_{n}-1} q_{r_{n}+s_{n}}-q_{r_{n}} q_{r_{n}+s_{n}-1}, q_{r_{n}-1} p_{r_{n}+s_{n}}-q_{r_{n}} p_{r_{n}+s_{n}-1},\right. \\
\left.p_{r_{n}-1} q_{r_{n}+s_{n}}-p_{r_{n}} q_{r_{n}+s_{n}-1}, p_{r_{n}-1} p_{r_{n}+s_{n}}-p_{r_{n}} p_{r_{n}+s_{n}-1}\right),
\end{array}
$$

it follows from (5.4), (5.5) and (5.6) that

$$
\prod_{1 \leq j \leq 4}\left|L_{j}\left(\underline{z}_{n}\right)\right| \ll q_{r_{n}}^{2} q_{r_{n}+s_{n}}^{2} q_{r_{n}+\left\lfloor w s_{n}\right\rfloor}^{-2} .
$$

Again on the assumption that $\left(q_{\ell}^{1 / \ell}\right)_{\ell \geq 1}$ converges, we are able to apply Theorem 3.1 (and, with some additional work, deduce that $\alpha$ is transcendental) only when

$$
\limsup _{n \rightarrow+\infty} \frac{r_{n}+w s_{n}}{2 r_{n}+s_{n}}>1
$$

that is, when

$$
w>w^{\prime}+1 \text {. }
$$

This is precisely the inequality (5.1) with $m=M$.

The novelty in [10] is the observation that the estimate (5.6) can be considerably improved when $r_{n}$ is large. Namely, using (5.2), (5.4), and (5.5), we get

$$
\begin{aligned}
\left|P_{n}(\alpha)\right| & \left|P_{n}(\alpha)-P_{n}\left(\alpha_{n}\right)\right| \\
= & \mid\left(q_{r_{n}-1} q_{r_{n}+s_{n}}-q_{r_{n}} q_{r_{n}+s_{n}-1}\right)\left(\alpha-\alpha_{n}\right)\left(\alpha+\alpha_{n}\right) \\
& -\left(q_{r_{n}-1} p_{r_{n}+s_{n}}-q_{r_{n}} p_{r_{n}+s_{n}-1}+p_{r_{n}-1} q_{r_{n}+s_{n}}-p_{r_{n}} q_{r_{n}+s_{n}-1}\right)\left(\alpha-\alpha_{n}\right) \mid \\
= & \left|\alpha-\alpha_{n}\right| \cdot \mid\left(q_{r_{n}-1} q_{r_{n}+s_{n}}-q_{r_{n}} q_{r_{n}+s_{n}-1}\right) \alpha-\left(q_{r_{n}-1} p_{r_{n}+s_{n}}-q_{r_{n}} p_{r_{n}+s_{n}-1}\right) \\
& \quad+\left(q_{r_{n}-1} q_{r_{n}+s_{n}}-q_{r_{n}} q_{r_{n}+s_{n}-1}\right) \alpha-\left(p_{r_{n}-1} q_{r_{n}+s_{n}}-p_{r_{n}} q_{r_{n}+s_{n}-1}\right) \\
& \quad+\left(q_{r_{n}-1} q_{r_{n}+s_{n}}-q_{r_{n}} q_{r_{n}+s_{n}-1}\right)\left(\alpha_{n}-\alpha\right) \mid \\
\ll & \left|\alpha-\alpha_{n}\right| \cdot\left(q_{r_{n}} q_{r_{n}+s_{n}}^{-1}+q_{r_{n}}^{-1} q_{r_{n}+s_{n}}+q_{r_{n}} q_{r_{n}+s_{n}}\left|\alpha-\alpha_{n}\right|\right) \\
\ll & \left|\alpha-\alpha_{n}\right| q_{r_{n}}^{-1} q_{r_{n}+s_{n}} \\
\ll & q_{r_{n}}^{-1} q_{r_{n}+s_{n}} q_{r_{n}+\left\lfloor w s_{n}\right\rfloor}^{-2} .
\end{aligned}
$$

Compared to the estimate (5.6), which was used in [1], we gain a factor $q_{r_{n}}^{-2}$. As we will see below, this allows us eventually to replace the assumption (5.8) by (5.11) below. The improvement can be explained by Lemma 4.1. Indeed, since

$$
\left|P_{n}(\alpha)\right| \leq H\left(P_{n}\right) \cdot\left|\alpha-\alpha_{n}\right| \cdot\left|\alpha-\alpha_{n}^{\prime}\right|,
$$


where $\alpha_{n}^{\prime}$ denotes the Galois conjugate of $\alpha_{n}$, we get an improvement on (5.6) when $\alpha_{n}^{\prime}$ is close to $\alpha$, that is, when $\alpha_{n}^{\prime}$ is close to $\alpha_{n}$. And Lemma 4.1 precisely asserts that this situation holds when $r_{n}$ is large.

Using (5.9) we can slightly improve (5.3) by applying the first statement of Corollary 3.2 instead of the second one. Indeed, we can conclude that $\alpha$ is transcendental when there exist $\varepsilon>0$ and arbitrarily large integers $n$ such that

$$
q_{r_{n}}^{-1} q_{r_{n}+s_{n}} q_{r_{n}+\left\lfloor w s_{n}\right\rfloor}^{-2} q_{r_{n}}^{2} q_{r_{n}+s_{n}}^{2}<\left(q_{r_{n}} q_{r_{n}+s_{n}}\right)^{-\varepsilon}
$$

If $\left(q_{\ell}^{1 / \ell}\right)_{\ell \geq 1}$ converges, this shows that the assumption

$$
w>w^{\prime}+3 / 2,
$$

is enough to deduce that $\alpha$ is transcendental.

By combining the use of Theorem 3.1 with $m=4$ and (5.9), we are able to improve (5.8) in the same way as (5.10) improves (5.3). Namely, we have

$$
\begin{aligned}
\prod_{1 \leq j \leq 4}\left|L_{j}\left(\underline{z}_{n}\right)\right| & \ll q_{r_{n}+s_{n}}^{2} q_{r_{n}+\left\lfloor w s_{n}\right\rfloor}^{-2} \\
& \ll 2^{-(w-1) s_{n}} \\
& \ll\left(q_{r_{n}} q_{r_{n}+s_{n}}\right)^{-\delta(w-1) s_{n} /\left(2 r_{n}+s_{n}\right)}
\end{aligned}
$$

if $n$ is sufficiently large, where we have set

$$
\delta=\frac{\log 2}{1+\lim \sup _{\ell \rightarrow+\infty} q_{\ell}^{1 / \ell}} .
$$

Thus, with $\varepsilon=\delta(w-1) /\left(2 w^{\prime}+2\right)$, which is positive when

$$
w>1,
$$

we see that

$$
\prod_{1 \leq j \leq 4}\left|L_{j}\left(\underline{z}_{n}\right)\right| \ll\left(q_{r_{n}} q_{r_{n}+s_{n}}\right)^{-\varepsilon}
$$

holds for any sufficiently large integer $n$.

We can then apply Theorem 3.1 to prove that $\alpha$ is transcendental. The details are given in [10].

\section{Two open questions}

We conclude this survey by two open questions.

Let $\left(a_{\ell}\right)_{\ell \geq 1}$ be the sequence defined by $a_{\ell}=2$ if $\ell$ is a perfect square, and $a_{\ell}=1$ otherwise. Is the real number

$$
\left[0 ; a_{1}, a_{2}, \ldots\right]=[0 ; 2,1,1,2,1,1, \ldots]
$$


transcendental? Theorem 2.3 cannot be applied in this case since the sequence of squares grows too slowly.

Theorem 2.2 asserts that automatic continued fractions are transcendental or quadratic. Conjecturally, the same holds for morphic continued fractions (see [8] for a precise definition). Since there exist morphic words $\mathbf{a}=\left(a_{\ell}\right)_{\ell \geq 1}$ whose complexity function $n \mapsto$ $p(n, \mathbf{a})$ grows as fast as a constant times $n^{2}$, Theorem 2.3 is not strong enough to give a positive answer to this conjecture.

\section{References}

[1] B. Adamczewski and Y. Bugeaud, On the complexity of algebraic numbers, II. Continued fractions, Acta Math. 195 (2005), 1-20.

[2] B. Adamczewski and Y. Bugeaud, On the complexity of algebraic numbers, I. Expansions in integer bases, Ann. of Math. 165 (2007), 547-565.

[3] B. Adamczewski and Y. Bugeaud, On the Maillet-Baker continued fractions, J. reine angew. Math. 606 (2007), 105-121.

[4] B. Adamczewski and Y. Bugeaud, Palindromic continued fractions, Ann. Inst. Fourier (Grenoble) 57 (2007), 1557-1574.

[5] B. Adamczewski, Y. Bugeaud, and L. Davison, Continued fractions and transcendental numbers, Ann. Inst. Fourier (Grenoble) 56 (2006), 2093-2113.

[6] B. Adamczewski, Y. Bugeaud et F. Luca, Sur la complexité des nombres algébriques, C. R. Acad. Sci. Paris 339 (2004), 11-14.

[7] J.-P. Allouche, J. L. Davison, M. Queffélec, and L. Q. Zamboni, Transcendence of Sturmian or morphic continued fractions, J. Number Theory 91 (2001), 39-66.

[8] J.-P. Allouche and J. Shallit, Automatic Sequences: Theory, Applications, Generalizations, Cambridge University Press, Cambridge, 2003.

[9] A. Baker, Continued fractions of transcendental numbers, Mathematika 9 (1962), $1-8$.

[10] Y. Bugeaud, Automatic continued fractions are transcendental or quadratic. Preprint.

[11] Y. Bugeaud, Continued fractions with low complexity: Transcendence measures and quadratic approximation, Compos. Math. 148 (2012), 718-750.

[12] A. Cobham, On the Hartmanis-Stearns problem for a class of tag machines. In: Conference Record of 1968 Ninth Annual Symposium on Switching and Automata Theory, Schenectady, New York (1968), 51-60.

[13] A. Cobham, Uniform tag sequences, Math. Systems Theory 6 (1972), 164-192. 
[14] J. L. Davison, A class of transcendental numbers with bounded partial quotients. In: Number theory and applications (Banff, AB, 1988), 365-371, NATO Adv. Sci. Inst. Ser. C Math. Phys. Sci., 265, Kluwer Acad. Publ., Dordrecht, 1989.

[15] P. Liardet et P. Stambul, Séries de Engel et fractions continuées, J. Théor. Nombres Bordeaux 12 (2000), 37-68.

[16] J. Liouville, Sur des classes très étendues de quantités dont la valeur n'est ni algébrique, ni même réductible à des irrationelles algébriques, C. R. Acad. Sci. Paris 18 (1844), 883-885; 910-911.

[17] J. H. Loxton and A. J. van der Poorten, Arithmetic properties of the solutions of a class of functional equations, J. reine angew. Math. 330 (1982), 159-172.

[18] J. H. Loxton and A. J. van der Poorten, Arithmetic properties of automata: regular sequences, J. reine angew. Math. 392 (1988), 57-69.

[19] E. Maillet, Introduction à la théorie des nombres transcendants et des propriétés arithmétiques des fonctions. Gauthier-Villars, Paris, 1906.

[20] M. Morse and G. A. Hedlund, Symbolic dynamics, Amer. J. Math. 60 (1938), $815-866$.

[21] M. Morse and G. A. Hedlund, Symbolic dynamics II, Amer. J. Math. 62 (1940), $1-42$.

[22] O. Perron, Die Lehre von den Kettenbrüchen, Teubner, Leibzig, 1929.

[23] M. Queffélec, Transcendance des fractions continues de Thue-Morse, J. Number Theory 73 (1998), 201-211.

[24] M. Queffélec, Irrational numbers with automaton-generated continued fraction expansion. In: Dynamical systems (Luminy-Marseille, 1998), 190-198, World Sci. Publ., River Edge, NJ, 2000.

[25] W. M. Schmidt, Norm form equations, Ann. of Math. 96 (1972), 526-551.

[26] W. M. Schmidt, Diophantine approximation, Lecture Notes in Mathematics 785, Springer, Berlin, 1980.

Yann Bugeaud

Université de Strasbourg

Mathématiques

7, rue René Descartes

67084 STRASBOURG (FRANCE)

bugeaud@math.unistra.fr 